\title{
Adherence to UK dietary guidelines is associated with higher dietary intake of total and specific polyphenols compared with a traditional UK diet: further analysis of data from the Cardiovascular risk REduction Study: Supported by an Integrated Dietary Approach (CRESSIDA) randomised controlled trial
}

\author{
Monica L. Castro-Acosta, Thomas A. B. Sanders, Dianne P. Reidlinger, Julia Darzi and Wendy L. Hall* \\ Diabetes \& Nutritional Sciences Division, King's College London, Franklin-Wilkins Building, 150 Stamford Street, London \\ SE1 $9 N H, U K$
}

(Submitted 19 June 2018 - Final revision received 10 October 2018 - Accepted 1 November 2018 - First published online 17 December 2018 )

\section{Abstract}

Adherence to dietary guidelines (DG) may result in higher intake of polyphenols via increased consumption of fruits, vegetables and whole grains. We compared polyphenol dietary intake and urinary excretion between two intervention groups in the Cardiovascular risk REduction Study: Supported by an Integrated Dietary Approach study: a 12-week parallel-arm, randomised controlled trial ( $n$ 161; sixty-four males, ninetyseven females; aged 40-70 years). One group adhered to UK DG, whereas the other group consumed a representative UK diet (control). We estimated polyphenol dietary intake, using a 4-d food diary (4-DFD) and FFQ, and analysed 24-h polyphenol urinary excretion by liquid chromatography-tandem MS on a subset of participants ( $n 46$ control; $n 45$ DG). A polyphenol food composition database for 4-DFD analysis was generated using Phenol-Explorer and USDA databases. Total polyphenol intake by 4-DFD at endpoint (geometric means with 95\% CI, adjusted for baseline and sex) was significantly higher in the DG group (1279 mg/d per $10 \mathrm{MJ} ; 1158,1412)$ compared with the control group $(1084 \mathrm{mg} / \mathrm{d}$ per $10 \mathrm{MJ}$; 980, 1197). The greater total polyphenol intake in the DG group was attributed to higher intake of anthocyanins, proanthocyanidins and hydroxycinnamic acids, with the primary food sources being fruits, cereal products, nuts and seeds. FFQ estimates of flavonoid intake also detected greater intake in DG compared with the control group. 24-h urinary excretion showed consistency with 4-DFD in their ability to discriminate between dietary intervention groups for six out of ten selected, individual polyphenols. In conclusion, following UK DG increased total polyphenol intake by approximately $20 \%$, but not all polyphenol subclasses corresponded with this finding.

Key words: Polyphenols: Dietary intake: Dietary guidelines: Randomised controlled trials

Greater consumption of fruits and vegetables (F\&V) and whole grains is associated with reduced risk of chronic diseases ${ }^{(1-5)}$. Increased intakes of different components present in these food groups, like fibre ${ }^{(6)}$, micronutrients ${ }^{(7)}$ and polyphenols ${ }^{(8)}$, have been identified as being partly responsible for beneficial effects. However, results from the UK National Diet and Nutrition Survey (NDNS) rolling programme ${ }^{(9-11)}$ reveal that consumption of F\&V and whole grains is below the recommended intake in at least $70 \%$ of the UK adult population ${ }^{(9-12)}$. Dietary polyphenols are a diverse range of phytochemicals containing one or more aromatic rings attached to a hydroxyl group. The term 'polyphenol' commonly encompasses phenolic acids, flavonoids, stilbenes and lignans, which are derived from a wide range of plant foods including fruits, vegetables and cereals. However, beverages such as tea, coffee, red wine and fruit juices represent the main dietary sources ${ }^{(13)}$. Epidemiological studies have shown a negative relationship between consumption of polyphenols and $\mathrm{CVD}^{(14-18)}$, cancer ${ }^{(19-22)}$ and type 2 diabetes ${ }^{(18,23-26)}$. Multiple mechanisms have been identified that may contribute to any direct causal relationship between dietary polyphenols and prevention of chronic diseases, including modulating inflammatory pathways, exerting effects on oxidative signalling and enzyme activity and regulation of gene expression ${ }^{(8)}$.

Previous reports in UK population, using either $24 \mathrm{~h}$ recall ${ }^{(27-31)}$ or food diaries ${ }^{(27-31)}$, suggest that habitual polyphenol intake is in the range of $800-1600 \mathrm{mg} / \mathrm{d}$, depending on the population studied. However, the use of different dietary assessment methods, food polyphenol composition databases and gaps in polyphenol composition data for certain foods limit the reliability of current intake estimates in various countries. ${ }^{(32)}$ Food diaries may provide a relatively accurate estimate of polyphenol intake and are more suitable for dietary intervention studies with smaller study population, but

Abbreviations: 4-DFD, 4-d food diary; CRESSIDA, Cardiovascular risk REduction Study: Supported by an Integrated Dietary Approach; DG, dietary guidelines; $\mathrm{F} \& \mathrm{~V}$, fruits and vegetables.

* Corresponding author: W. L. Hall, email wendy.hall@kcl.ac.uk 
they reflect short-term intake rather than habitual consumption patterns, which could be particularly misleading for seasonally available foods ${ }^{(33)}$. Research groups have designed and validated FFQ to estimate dietary flavonoid intake in different populations $^{(34,35)}$, which may provide more reliable habitual intake estimations for specific population. However, these remain unavoidably susceptible to bias due to the self-reporting errors, portion size quantification and estimation errors resulting from the lack of data on polyphenol content in food ${ }^{(36)}$. Whichever dietary assessment method is selected, the resulting intake data will only be accurate if the polyphenol composition of food database is fit for purpose. At present, the most commonly used food polyphenol composition databases are Phenol-Explorer ${ }^{(37-39)}$, which provides information on the content of 502 polyphenols (of the four classes) in 459 food items including aglycones, glycosides and esters and the USDA databases ${ }^{(40-42)}$ which includes thirty-five flavonoids (aglycones only) in 506 food items, respectively. Urinary excretion of polyphenols has been shown to be a suitable biomarker for the intake of polyphenols ${ }^{(43,44)}, \quad F \& V^{(45,46)}$, polyphenol-rich beverages $^{(47,48)}$ and polyphenol-rich food ${ }^{(49,50)}$. The presence of polyphenol metabolites in urine is closely related to the quantity consumed and the overall metabolism in the body. However, different polyphenols can produce common metabolites, so the biomarker selected must reflect the specific intake of the parent polyphenol in question to accurately estimate the intake of individual phenolic compounds ${ }^{(51)}$.

Dietary guidelines in the UK are population-based recommendations for maintenance of health and well-being, and to reduce risk of chronic diseases. No dietary recommendations exist for polyphenols, although the advantages and disadvantages of this approach have been debated ${ }^{(52-54)}$. A dietary pattern consistent with current dietary guidelines (DG) could reasonably be assumed to be richer in polyphenols than the average UK dietary pattern due to increased intake of F\&V and whole grains. However, not all fruits and vegetables are polyphenol rich ${ }^{(55)}$, and in fact the majority of dietary polyphenols in the UK are provided by tea, coffee and cocoa intake ${ }^{(30)}$. The aim of this study was to compare dietary polyphenol intake in a free-living study population randomised to either following UK DG or consuming a representative, more traditional UK diet. The hypothesis was that adherence to UK DG would result in an increase in total dietary polyphenol intake compared with a diet that was more representative of the UK adult population. The primary outcome variable was total polyphenol intake, adjusted for energy intake, assessed by a 4-d food diary (4-DFD). Secondary outcome variables included intake of classes, subclasses and individual phenolic acids/polyphenols. Data were also compared with $\mathrm{FFQ}^{(15)}$ and biomarkers of polyphenol intake ( $24 \mathrm{~h}$ excretion of representative urinary metabolites)

\section{Methods \\ Study design}

The Cardiovascular risk REduction Study: Supported by an Integrated Dietary Approach (CRESSIDA) was a 12-week parallel-designed, randomised, controlled trial funded by the
Food Standards Agency/Department of Health (UK) (N02047) and sponsored by King's College London. This study was conducted according to the guidelines laid down in the Declaration of Helsinki, and all procedures involving human subjects were approved by the St Thomas' Hospital Research Ethics Committee $(10 / \mathrm{H} 0802 / 24)^{(56)}$. Written informed consent was obtained from all subjects. This trial was registered at current controlled trial (http://www.controlled-trials.com/ ISRCTN) as ISRCTN92382106. The primary aim of the original study $^{(56)}$ was to assess the effects on CVD risk factors when following a diet based on UK DG in comparison to a representative UK diet (control). A sample size of 78/group had $90 \%$ power to detect a $4 \mathrm{mmHg}$ effect of diet on daytime systolic ambulatory blood pressure $(\alpha=0.05)$; this sample size was also large enough to detect a $5 \%$ change in the ratio of total cholesterol: HDL-cholesterol and a $1 \%$ unit change in flowmediated dilatation of the brachial artery with sufficient statistical power. The results of these primary outcomes have been published previously ${ }^{(56)}$.

The data presented here comprise a secondary analysis of dietary intake data from both the DG and control groups at baseline and endpoint of the 12-week dietary intervention period, to determine whether adherence to UK DG results in an increase in polyphenol intake.

\section{Participants}

Healthy men and women (BMI $\geq 18.5$ and $\leq 35 \mathrm{~kg} / \mathrm{m}^{2}$ ) were recruited from the London area (August 2010-July 2012) by newspaper (London Metro) and electronic advertisement (King's College London email and website). Participants between the age of 40-70years were chosen because risk increases markedly with age and an upper age limit of 70 years was selected because absolute annual risk of CVD exceeds $2 \%$ beyond that age in the majority of people, and a higher proportion are receiving medication on daily basis. A full list of inclusion and exclusion criteria have been reported previously $^{(56)}$. For randomisation, a purpose-designed clinical database was used (MedSciNet AB), which undertook the minimisation randomisation, balancing the treatment arms for the minimisation variables of sex, age and ethnicity ${ }^{(56)}$. Participants received dietary advice in person at baseline and at week 4 , and by phone at weeks 6 and 8. Dietary assessment included two FFQ and two 4-DFD administered at baseline and endpoint and two 24-h recalls administered at weeks 4 and 8 . Urine samples were collected at four time points: baseline, week 4 , week 8 and endpoint.

\section{Dietary advice}

Participants randomised to the DG group were advised to increase the intake of $\mathrm{F} \& \mathrm{~V}$ to 5 portions/d; intake of whole grains to $>50 \%$ of cereal intake; to consume 2 portions of fish/ week (one of which should be oily); to replace full fat with reduced fat dairy products; to replace fats rich in SFA with spreads/oils low in SFA and high in MUFA; to restrict salt intake to $<6 \mathrm{~g} / \mathrm{d}(<100 \mathrm{mmol} / \mathrm{d})$ and to reduce intake of free sugars. The control diet comprised a nutritionally balanced, traditional 
UK diet, formulated with familiar foods (full cream milk, cheese, butter, meat and meat-products, non-wholegrain cereals), reflecting typical UK intake of $\mathrm{F} \& \mathrm{~V}$ (three portions/d), with a higher content of SFA (14\% energy), unrestricted intake of salt and sugar and low intake of oily fish. The study included provision of spread, oil, whole grain pasta, rice and cereal bars, minimally processed wholegrain breakfast cereal (oats, muesli, etc.), almonds and macadamia nuts and tinned oily fish to the intervention group and spread, oil and refined cereals as pantry items to the control group. Participants of both groups were instructed to refrain from taking nutritional supplements during the study ${ }^{(57)}$.

\section{4-d food diary}

Dietary intake of polyphenols was quantified from 4-DFD, at baseline and endpoint of the 12-week intervention. A polyphenol food composition database was generated using Phenol-Explorer ${ }^{(37-39)}$ and USDA database ${ }^{(40-42)}$ and extended using polyphenol retention factors and recipes provided by participants. If a recipe was not provided by a participant, a standard recipe was obtained from the UK food tables ${ }^{(58)}$, a UK food industry recipe book ${ }^{(59)}$, the BBC Good Food website ${ }^{(60)}$ or additional websites specialised in UK recipes and other countries' typical cuisines. In total, 118 recipes were obtained from participants, ninety-three from UK food tables, twenty from a UK food industry recipe book, seventy-eight from the BBC Good Food website and ninety-four from other websites. Polyphenol intake was estimated for four classes of polyphenols: flavonoids, phenolic acids, lignans and stilbenes. An additional group of polyphenols were included under the name 'other polyphenols'; since Phenol-Explorer grouped a series of compounds including alkylmethoxyphenols, alkylphenols, curcuminoids, furanocoumarins and tyrosols. The flavonoid intake was analysed in eight subclasses: anthocyanins, dihydrochalcones, flavanols (flavan-3-ols monomers and theaflavins), proanthocyanidins, flavanones, flavones, flavonols and isoflavones, for each subclass a range of 2-11 of the most representative individual compounds were selected. For phenolic acids, subclass two groups were analysed: hydroxybenzoic acids and hydroxycinnamic acids, for each group a range of 4-6 of the most representative individual compounds were selected. For lignans subclass, four individual compounds were analysed: pinoresinol, lariciresinol, secoisolariciresinol and matairesinol. For stilbenes subclass, resveratrol was selected and analysed. A total of fifty-two individual polyphenols were analysed and 1141 food items were included in the final database.

\section{FFQ}

Dietary intake of flavonoids was quantified from FFQ (EPICNorfolk FFQ version 6) ${ }^{(61)}$ at baseline and endpoint of the 12 -week intervention. Flavonoid intake was estimated from six subclasses and the most representative compounds for each: flavanones (hesperetin, naringenin, eriodictyol), anthocyanins (cyanidin, delphinidin, malvidin, pelargonidin, peonidin, petunidin), flavones (apigenin, luteolin), flavonols (quercetin, kaempferol, myricetin, isorhamnetin), flavan-3-ols (catechin, epicatechin, gallocatechin, epigallocatechin, epicatechin-3-gallate, epigallocatechin-3-gallate) and polymers (theaflavins, thearubigins and proanthocyanidins). In total, thirty-two individual flavonoids were analysed using a database created and kindly provided by Dr Amy Jennings and Professor Aedin Cassidy from the University of East Anglia ${ }^{(15)}$ and modified for the requirements of the FFQ analysis, so that the flavonoid content of 130 food items was analysed. FFQ registers the frequency of consumption of specific foods, of standard portion size, in a month. There were nine answer options that varied from 'never or less than once in a month' to 'six or more per day'. To calculate the total intake of subclasses of flavonoids, frequencies were converted to daily portions and multiplied by flavonoid content in each specific food item; the results were summed for each subclass and for each participant.

\section{Liquid chromatography-tandem MS}

24-h urine collections were made as previously described ${ }^{(56)}$, using boric acid as preservative ${ }^{(62)}$ and aliquots stored at $-80^{\circ} \mathrm{C}$ until analysis. Completeness of urine collection was measured using recovery of para-aminobenzoic acid, according to a standard protocol $^{(63)}$. Liquid chromatography-tandem MS analysis was only performed on endpoint urine samples collected at week 12 of the intervention to coincide with the dietary assessment by 4-DFD. A previously published protocol for polyphenol analysis in urine ${ }^{(47)}$ was adapted and developed for the quantification of ten aglycone metabolites of polyphenols. A subsample of the CRESSIDA study was selected for the analysis; the selection was based on the intake of F\&V reported in the endpoint FFQ, subsample included participants in the control group reporting the lowest intake of $F \& V$ and participants in the intervention group reporting the highest intake of F\&V were analysed. Quantification of endpoint samples allowed the comparison between groups at the end of the dietary intervention; the aim was to detect greater amounts of polyphenols excreted in urine by participants who reported consuming the most polyphenols by FFQ compared with participants who reported consuming the least and to determine the agreement with food diary data. One or two of the most representative aglycones of different polyphenol subclasses was selected for quantification. A total of ten phenolic compounds were analysed: phloretin (dihydrochalcones), epicatechin (flavan-3-ols), hesperetin and eriodictyol (flavanones), luteolin (flavones), quercetin (flavonols), daidzein (isoflavones), gallic and vanillic acid (phenolic acids) and enterolactone as a product of microbial metabolism of lignans in colon. The phenolic compounds selected for quantification have been suggested as suitable biomarkers of polyphenol intake ${ }^{(55)}$, with a high recovery and good correlation with F\&V intake when estimated by FFQ, $24 \mathrm{~h}$ recalls or food diaries ${ }^{(50)}$. The major food sources of the ten phenolic compounds are $\mathrm{F} \& \mathrm{~V}$ and wholegrain products, or represent the main metabolites of parent glucosides present in the previously mentioned foods.

Purified standards phloretin, epicatechin, eriodictyol, hesperetin, luteolin, daidzein, gallic and vanillic acids were obtained from Santa Cruz Biotechnology, and quercetin, enterolactone and catechin-2,3,4- ${ }^{13} \mathrm{C}_{3}$ from Sigma-Aldrich. Stock solutions were prepared for all the purified phenolic 
compounds, $2 \mathrm{mg}$ of each of the ten purified compounds was diluted in $1 \mathrm{ml}$ of methanol or dimethyl sulphoxide, according to supplier specifications and $1 \mathrm{mg}$ of catechin-2,3,4- ${ }^{13} \mathrm{C}_{3}$ (internal standard) in $1 \mathrm{ml}$ of methanol. Stock solutions were stored at -40 or $-80^{\circ} \mathrm{C}$ (internal standard). Enzyme $\beta$-glucuronidase/sulphatase $(0.05 \mathrm{~g})$ type $\mathrm{H}-5$ from Helix pomatia (SigmaAldrich) was diluted in $1 \mathrm{ml}$ of $0.2 \%$ sodium chloride solution to create a working solution of 50000 units $/ \mathrm{ml}$; the enzyme solution was stored at $-40^{\circ} \mathrm{C}$. Urine samples $(250 \mu \mathrm{l})$ were processed for hydrolysis of glucuronide and sulphated metabolites using a modified version of Ito et al. ${ }^{(47)}$. Urine samples were acidified with acetic acid ( $20 \mu \mathrm{l}, 0.58 \mathrm{~m})$ and incubated with 1300 units of $\beta$-glucuronidase/sulphatase and $300 \mathrm{ng}$ of internal standard at $37^{\circ} \mathrm{C}, 120 \mathrm{rpm}$ for $1.5 \mathrm{~h}$. A liquid-liquid extraction with ethyl acetate was performed twice (400 and $300 \mu \mathrm{l})$. The two organic layers were pooled and evaporated to dryness under $\mathrm{N}_{2}$ then stored at $-80^{\circ} \mathrm{C}$ for $1-4 \mathrm{~d}$ until their reconstitution with $100 \mu \mathrm{l}$ of $40 \%$ methanol immediately before injection. Analysis of urine samples was performed on a HPLC system Hewlett-Packard series 1100 binary pump, coupled with a triple quadrupole mass spectrometer, Micromass Quattro LC (Micromass Limited), operating in negative electrospray ionisation (ES-) mode, equipped with a Zorbax SB-C18 column $(2 \cdot 1 \times 50$, $3.5 \mathrm{~mm}$; Agilent). Ionisation and fragmentation were optimised for each polyphenol by direct infusion of a standard solution, and specific values for collision energy parameters were identified for each polyphenol. Peak for each polyphenol identity was established by the parent and daughter ion pair peak and retention time. Solvents A (water with $0.1 \% \mathrm{v} / \mathrm{v}$ formic acid) and $\mathrm{B}$ (acetonitrile with $0 \cdot 1 \% \mathrm{v} / \mathrm{v}$ formic acid) were run in a $95 /$ $5 \%$ proportion at a flow rate of $0.2 \mathrm{ml} / \mathrm{min}$ with the following gradient: $0-1 \mathrm{~min}, 5 \%$ solvent $\mathrm{B} ; 1-4 \mathrm{~min}$, increase solvent $\mathrm{B}$ from 5 to $10 \%$; $4-5 \mathrm{~min}$, increase solvent B from 10 to $90 \%$, 5-5.2 min, decrease solvent B from 90 to 5\%, 5.2-15 min isocratic for $9.8 \mathrm{~min}$. Peak areas were plotted against the internal standard response. A good linearity ( $r^{2}$ 0.970-0.990) was observed for all the polyphenols quantified except for luteolin $\left(r^{2}\right.$ 0.922). Samples were run in the same batch and chromatograms were processed automatically by MassLynx Mass Spectrometry Software (MASSLYNX ${ }^{\mathrm{TM}}$ version 3.5) using the same processing integrate parameters, peak-to-peak amplitude and peak detection. Intra-assay co-efficients of variability were phloretin $7.5 \%$, epicatechin $6 \%$, hesperetin $21.9 \%$, eriodictyol $9.6 \%$, luteolin $9 \cdot 8 \%$, quercetin $10 \cdot 8 \%$, daidzein $3.1 \%$, gallic acid $21.4 \%$, vanillic acid $14.4 \%$ and enterolactone $5 \cdot 1 \%$. Final quantities of phenolics compounds were estimated after adjusting the urinary volume. Calibration curves were prepared by spiking HPLC-grade water with $2 \cdot 5,5,12 \cdot 5,25,50,100,150,200$ and $250 \mu$ of mixed polyphenols working solution and $100 \mu$ of internal standard working solution, the calibration curve range was $5-500 \mathrm{ng} / \mathrm{ml}$. Spiked HPLC-grade water samples were treated with enzyme and extracted with ethyl acetate as were the urine samples, and spiked samples were injected in duplicate at each concentration level. Limits of quantification were established using the spiked samples for calibration curves; minimum detected values were $5 \mathrm{ng} / \mathrm{ml}$ for phloretin, epicatechin, eriodictyol, hesperetin, luteolin, daidzein, gallic acid and vanillic acid and $10 \mathrm{ng} / \mathrm{ml}$ for quercetin and enterolactone.

\section{Statistical analysis}

Statistical analysis was performed using Statistical Package for the Social Sciences version 21. 4-DFD intake data ${ }^{(64)}$ were expressed as weight of intake $(\mathrm{g}) / \mathrm{d}$ per $10 \mathrm{MJ}$ energy intake, to adjust for the variability in total food intake between individuals over the $4 \mathrm{~d}$, whereas FFQ data were expressed simply as weight of intake $(\mathrm{g}) / \mathrm{d}$. Normality of data distribution was evaluated visually by inspection of the histograms and normal Q-Q plots. Non-normally distributed variables were natural log transformed before statistical analysis by parametric methods, or analysed by non-parametric methods where log transformation failed to yield a normal distribution. Independentsamples $t$ tests were conducted to compare groups at baseline to verify whether the polyphenol intake of each treatment group was similar before the dietary intervention. In cases where the data were normally distributed, one-way ANOVA was conducted, with sex as a fixed factor and baseline value as covariate (ANCOVA) was conducted to find differences between groups at endpoint. In instances where the data could not be transformed to a normal distribution, a Mann-Whitney $U$ test was conducted to find the differences between groups at endpoint. Correlation analyses on urinary and dietary polyphenols were conducted by two-tailed Spearman's correlations.

\section{Results}

A total of 165 healthy men and women aged $40-70$ years were recruited, and 162 completed the CRESSIDA study (sixty-four males, ninety-seven females) (Fig. 1). Dietary intake of polyphenols was quantified from $322 \times 4$-DFD $(n$ 161) and from 322 FFQ ( $n$ 161) (EPIC-Norfolk FFQ version 6$)^{(61)}$ at baseline and endpoint of the 12-week intervention, one participant randomised to the DG group did not complete the collection of food diaries and, therefore, was eliminated from the analysis. Urinary excretion of polyphenols was analysed in a subsample ( $n$ 91) of the CRESSIDA study: forty-five samples from participants in the control group reporting the lowest intake of $F \& V$, and forty-six samples from participants in the intervention group reporting the highest intake of $F \& V$.

Characteristics of the whole CRESSIDA study population at baseline are shown in Table 1. Mean BMI was significantly higher in the control group. Intakes of wholegrain cereals, fruits and vegetables and dietary fibre were higher in the study population at baseline than those reported in a larger, representative UK population sample ${ }^{(11,56)}$. As reported previously, at endpoint, the urinary $\mathrm{K}$ excretion was $9 \mathrm{mmol} / \mathrm{d}$ high in the DG group, indicating higher consumption of F\&V. Reported wholegrain cereal intake was $81 \mathrm{~g} / \mathrm{d}$ at endpoint in the DG group (mainly wheat, oats and rice) compared with the $32 \mathrm{~g} / \mathrm{d}$ in the control group, as confirmed by the high plasma alkylresorcinol concentrations, reflecting intake of whole grains mainly from wheat, barley and rye ${ }^{(56)}$.

\section{4-d food diary}

Median total polyphenol intake at baseline in the whole study population (with lower and upper limits of IQR) was $1183 \mathrm{mg} / \mathrm{d}$ $(745,1613)$, or $1282 \mathrm{mg} / \mathrm{d}$ per $10 \mathrm{MJ}$ energy intake $(896,1838)$. 


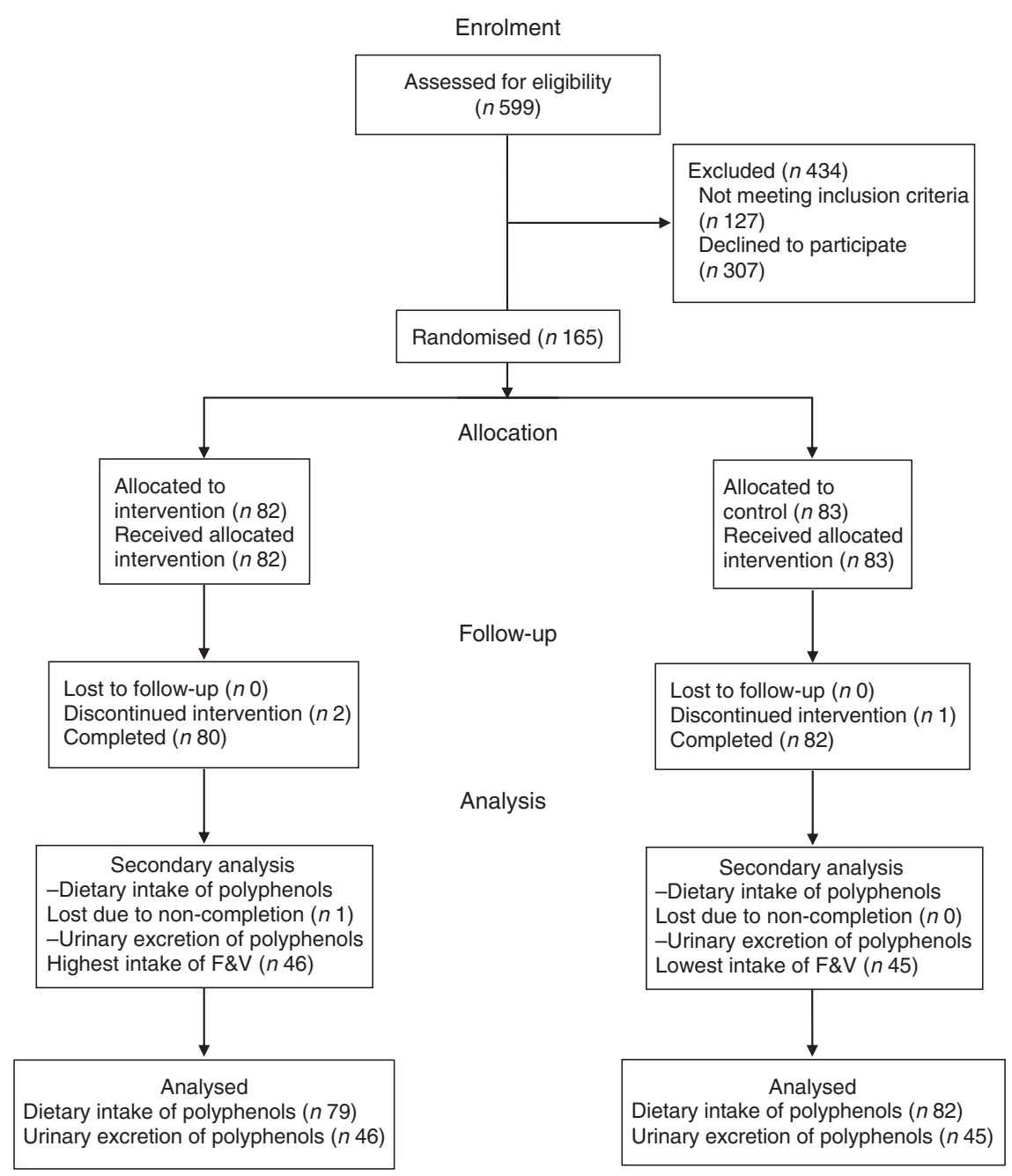

Fig. 1. Flow of participants through the Cardiovascular risk REduction Study: Supported by an Integrated Dietary Approach study. F\&V, fruits and vegetables.

Table 1. Baseline characteristics of the Cardiovascular risk REduction Study: Supported by an Integrated Dietary Approach study population by randomised group

(Numbers, mean values and standard deviations)

\begin{tabular}{|c|c|c|c|c|c|}
\hline \multirow[b]{2}{*}{ Characteristics } & \multicolumn{2}{|c|}{ Control ( $n$ 82) } & \multicolumn{2}{|c|}{ Dietary guidelines ( $n$ 79) } & \multirow[b]{2}{*}{ Difference between groups $\left(P^{\star}\right)$} \\
\hline & Mean & SD & Mean & SD & \\
\hline \multicolumn{6}{|l|}{$\operatorname{Sex}(n)$} \\
\hline Female & \multirow{2}{*}{\multicolumn{2}{|c|}{50}} & \multicolumn{2}{|c|}{47} & \\
\hline Male & & & \multicolumn{2}{|c|}{32} & \\
\hline Age (years) & 52 & 8.0 & 53 & $8 \cdot 0$ & 0.717 \\
\hline BMI $\left(\mathrm{kg} / \mathrm{m}^{2}\right)$ & $26 \cdot 8$ & 3.9 & 25.4 & 3.7 & 0.019 \\
\hline Waist circumference $(\mathrm{cm})$ & & & & & 0.093 \\
\hline Female & 91.9 & $10 \cdot 0$ & $87 \cdot 1$ & $12 \cdot 2$ & \\
\hline Male & $98 \cdot 2$ & $12 \cdot 2$ & $97 \cdot 3$ & $9 \cdot 3$ & \\
\hline $\mathrm{SBP}(\mathrm{mmHg})$ & $122 \cdot 0$ & 11.5 & 121.6 & $14 \cdot 3$ & 0.848 \\
\hline $\mathrm{DBP}(\mathrm{mmHg})$ & 73.9 & $7 \cdot 1$ & 73.8 & 8.0 & 0.930 \\
\hline Glucose $(\mathrm{mmol} / \mathrm{l})$ & $5 \cdot 0$ & 0.4 & $5 \cdot 3$ & 0.5 & 0.990 \\
\hline Total cholesterol (mmol/l) & $5 \cdot 3$ & 0.9 & 5.4 & 1.1 & 0.983 \\
\hline TC:HDL-cholesterol ratio & 3.6 & 1.0 & 3.5 & 1.0 & 0.262 \\
\hline
\end{tabular}

SBP, systolic blood pressure; DBP, diastolic blood pressure.

* Statistical comparisons between groups at baseline by independent $t$ test or independent-samples Mann-Whitney $U$ test. 
Table 2. Daily intake of polyphenols estimated by a 4-d food diary and adjusted for energy intake following dietary guidelines and control diets (Medians and interquartile ranges (IQR); geometric mean values and $95 \%$ confidence intervals)

\begin{tabular}{|c|c|c|c|c|c|c|c|}
\hline \multirow{3}{*}{$\begin{array}{l}\text { Polyphenols (mg/d per } 10 \mathrm{MJ} \text { ) } \\
\text { Classes and subclasses }\end{array}$} & & & \multicolumn{4}{|c|}{ Endpoint ( $n$ 161) } & \multirow{3}{*}{$\begin{array}{l}\text { Differences between } \\
\text { groups at endpoint }(P \dagger)\end{array}$} \\
\hline & \multicolumn{2}{|c|}{ Baseline ( $n$ 161) } & \multicolumn{2}{|c|}{ Control ( $n$ 82) } & \multicolumn{2}{|c|}{ Dietary guidelines ( $n$ 79) } & \\
\hline & Median & IQR & Median & IQR & Median & IQR & \\
\hline Total polyphenols $\ddagger$ & \multirow{2}{*}{\multicolumn{2}{|c|}{$1202^{*}$}} & & & & & $0.023 \S$ \\
\hline Mean & & & \multicolumn{2}{|c|}{1084} & \multicolumn{2}{|c|}{1279} & \\
\hline $95 \% \mathrm{Cl}$ & \multicolumn{2}{|c|}{1093,1323} & \multicolumn{2}{|c|}{980,1197} & \multicolumn{2}{|c|}{1158,1412} & \\
\hline Flavonoids & $840^{*}$ & 536,1263 & 802 & 456,1109 & 882 & 610,1401 & 0.014 \\
\hline Anthocyanins & 55 & 14,130 & 30 & 14,108 & 76 & 25,144 & 0.032 \\
\hline Dihydrochalcones & 1.6 & $0.0,3 \cdot 8$ & 2 & 0,4 & 2 & 1,6 & 0.297 \\
\hline Flavanols & 349 & 114,526 & 311 & 104,498 & 394 & 128,665 & 0.159 \\
\hline Proanthocyanidins & $231^{*}$ & 117,339 & 195 & 115,324 & 249 & 169,381 & 0.020 \\
\hline Flavanones & 31 & 4,64 & 24 & 2,58 & 33 & 8,84 & 0.096 \\
\hline Flavones & 6 & 3,12 & 5 & 1,12 & 5 & 2,12 & 0.603 \\
\hline Flavonols & 80 & 51,112 & 80 & 50,105 & 83 & 58,127 & 0.125 \\
\hline Isoflavones & 0.3 & $0.1,9.9$ & 0.1 & $0.1,0.4$ & 0.5 & $0 \cdot 1,65 \cdot 0$ & 0.002 \\
\hline Lignans & 0.5 & $0 \cdot 2,5 \cdot 0$ & 0.3 & $0.1,0.4$ & 0.5 & $0 \cdot 2,6 \cdot 0$ & $<0.001$ \\
\hline Phenolic acids & 289 & 185,569 & 221 & 132,493 & 344 & 209,522 & 0.012 \\
\hline Hydroxybenzoic acids & 90 & 35,143 & 75 & 32,125 & 100 & 55,170 & 0.067 \\
\hline Hydroxycinnamic acids & 149 & 88,450 & 119 & 59,378 & 172 & 99,444 & 0.018 \\
\hline Stilbenes & 0.09 & $0.02,0.71$ & 0.09 & $0.02,0.94$ & 0.26 & $0.07,1.25$ & 0.042 \\
\hline Other polyphenols & 21 & 11,41 & 13 & 6,30 & 37 & 20,58 & $<0.001$ \\
\hline
\end{tabular}

* Statistically significant differences between groups at baseline by independent $t$ test (total polyphenols) or independent-samples Mann-Whitney $U$ test (all other comparisons); intake was higher in the dietary guidelines group than the control group $(P<0.05)$.

† Statistical comparisons between groups at endpoint by Mann-Whitney $U$ test.

$\ddagger$ Adjusted for baseline and sex at endpoint.

$\S$ Statistical comparisons between groups at endpoint adjusted for baseline and sex by ANCOVA.

As shown in Table 2, baseline intake of total polyphenols was significantly higher in the DG group compared with the control group due to the high intake of total flavonoids, the main contributor being total proanthocyanidins

At endpoint, the total polyphenol intake per $10 \mathrm{MJ}$ energy intake, adjusted for sex and baseline polyphenol intake, was higher in the DG group than in the control group (Table 2). Analysis of intake of polyphenol classes at endpoint (unadjusted for sex and baseline, as non-parametric statistical analysis was necessary) demonstrated that the DG group had higher intake of total flavonoids (although also higher in DG at baseline), total lignans, phenolic acids, stilbenes and 'other polyphenols' compared with the control group. For the major flavonoid subclasses, the DG group reported higher intake of anthocyanins, proanthocyanidins (although these were also higher at baseline) and isoflavones but not dihydrochalcones, flavanols, flavones or flavonols. For the major phenolic acid subclasses, the DG group reported higher intake of hydroxycinnamic acids, but the trend for higher intake of hydroxybenzoic acids was not statistically significant.

For individual (poly)phenols, there were no differences between groups at baseline, but at endpoint the group following the DG diet reported higher intake of a number of anthocyanins including cyanidin, malvidin, peonidin and petunidin $(P<0.05)$ compared with the control group. Furthermore, intakes of individual flavanones (naringenin, eriodictyol and hesperetin, $P<0.05$ ), a flavone (luteolin, $P<0.05$ ) and isoflavones (daidzein and genistein, $P<0.01$ ) were higher in the DG group compared with the control group at endpoint. With regard to lignans, phenolic acids and stilbenes classes, there were higher intakes of secoisolariciresinol $(P<0.05)$, matairesinol $(P<0 \cdot 001)$, protocatechuic acid $(P<0 \cdot 01)$, vanillic acid $(P<0.001)$, 4-hydroxybenzoic acid $(P<0.001)$, syringic acid $(P<0.001), p$-coumaric acid $(P<0.001)$ and ferulic acid $(P<0.05)$ at endpoint in the DG group compared with the control group. There were no differences between groups in individual flavonols (including kaempferol, quercetin, myricetin and isorhamnetin) nor flavanols (including individual catechins and theaflavins).

The main sources of total polyphenols at endpoint in both groups were tea, coffee and wine (control: 61\%, DG: 59\%), fruits (control: 10\%, DG: 17\%) and fruit juices (control: $8 \%$, DG: $4 \%$ ) (Fig. 2). Main sources of flavonoids in control and DG groups were tea, coffee and wine (control: $55 \%$, DG: $55 \%$ ), fruits (control: 14\%, DG: $21 \%$ ) and fruit juices (control: $10 \%$, DG: $5 \%$ ). Main sources of the subclass flavanols subclass were tea, coffee and wine (control: 64\%, DG: 65\%), fruits (control: 13\%, DG: 19\%) and chocolates (control: $11 \%$, DG: $4 \%$ ). There was an increase in the percentage of polyphenols sourced by fruits, cereal products (breakfast cereal and cereal bar) and nuts and seeds (see Fig. 3 for further details) in the group following the DG diet compared with the baseline. There was a decrease in the percentage of polyphenols derived from the less recommended food options as fruit juices, chocolates and biscuits (Fig. 3).

\section{FFQ}

There was no difference in total flavonoids and flavonoid subclass estimated intake between groups at baseline. At endpoint, the DG group reported higher intake of total flavonoids, total anthocyanins, total flavones, total flavanols and total 

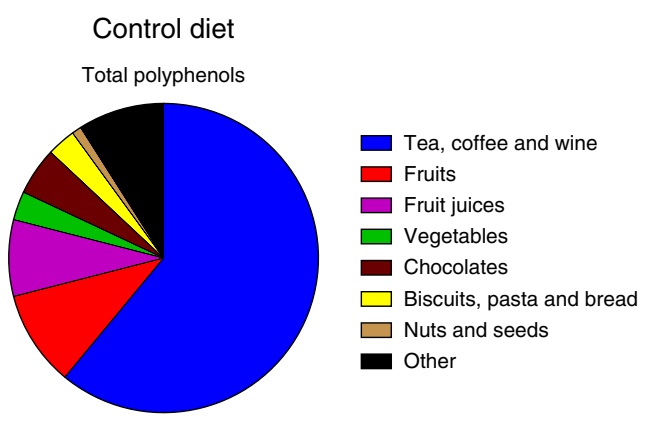

Dietary guideline diet
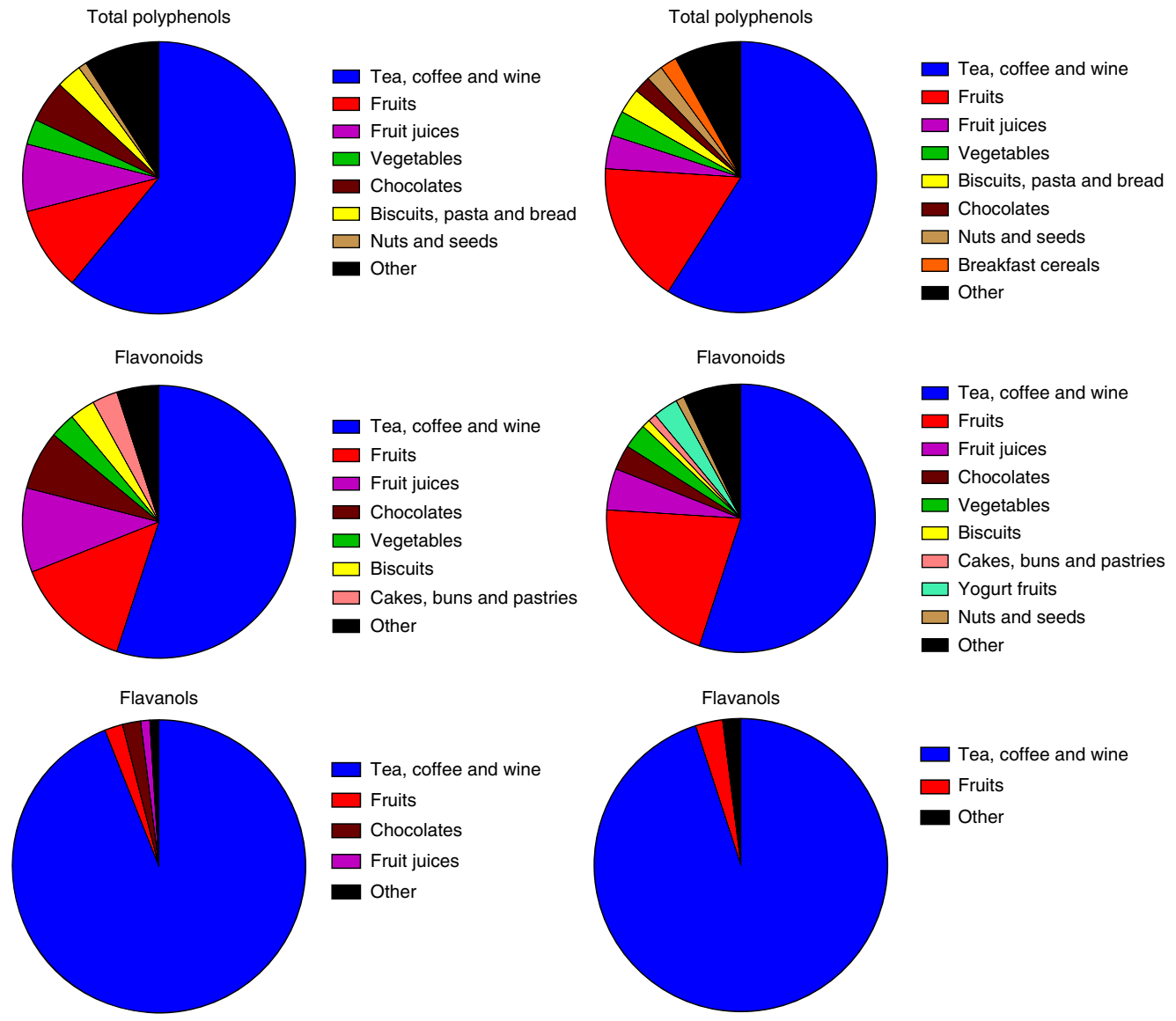

Fig. 2. Main polyphenol food sources in control and dietary guidelines groups, at endpoint, of the Cardiovascular risk REduction Study: Supported by an Integrated Dietary Approach study as estimated by a 4-d food diary.

proanthocyanidins (Table 3). Flavanone and flavonol intake did not differ between groups at endpoint. Differences between individual polyphenol components revealed higher reported intakes of cyanidin, delphinidin, petunidin and peonidin $(P<0.001)$, malvidin and pelargonidin $(P<0.005)$, catechin $(P<0.01)$, and epicatechin-3-O-gallate, epigallocatechin-3-Ogallate, myricetin and luteolin $(P<0.05)$ in the DG group (data not shown) compared with control.

\section{Urinary polyphenol excretion}

The ten aglycones quantified in urine were either metabolites of parent glucuronide/sulphated compounds formed in the human tissues or metabolites produced by the gut microbiota, for example, enterolactone from lignans present in fibre-rich food. At endpoint, there was a greater $24 \mathrm{~h}$ urinary excretion of phloretin, eriodictyol, hesperetin, luteolin, quercetin, gallic acid, vanillic acid and enterolactone in the DG group compared with the control group (Table 4), and a non-significant tendency towards a similar response for daidzein excretion. Daidzein was not detected in thirty-seven participants of the ninety-one subsample, which could contribute to the lack of significance in the results, given that values observed are considerably higher in the intervention group. The only polyphenol that was excreted in similar amounts in both dietary groups was epicatechin.

\section{Discussion}

The aim of this secondary analysis of dietary intake data from the CRESSIDA trial was to investigate whether following advice to adhere to UK DG increased polyphenol intake. Baseline polyphenol/flavonoid intake from 4-DFD and FFQ was in alignment with the previous reports in the UK population using $24 \mathrm{~h}$ recall ${ }^{(27-31,65-67)}$ or $\mathrm{FFQ}^{(15)}$. The hypothesis was supported by the finding that the total estimated polyphenol intake was approximately $200 \mathrm{mg} / 10 \mathrm{MJ}$ energy intake higher at endpoint in the DG group compared with the control group. However, estimates of total polyphenol intake are only as accurate as the polyphenol composition data and rely on the sum of the components, which may lead to underestimation if complete composition profile data are unavailable for individual foods. Therefore, total polyphenol intake data should be interpreted in the context of the individual polyphenol intake. Estimated dietary intake by 4-DFD showed that the DG group had higher intake of individual polyphenols/phenolic acids, where the main food sources were fruits and vegetables (cyanidin, malvidin, peonidin, petunidin, protocatechuic acid, naringenin, 


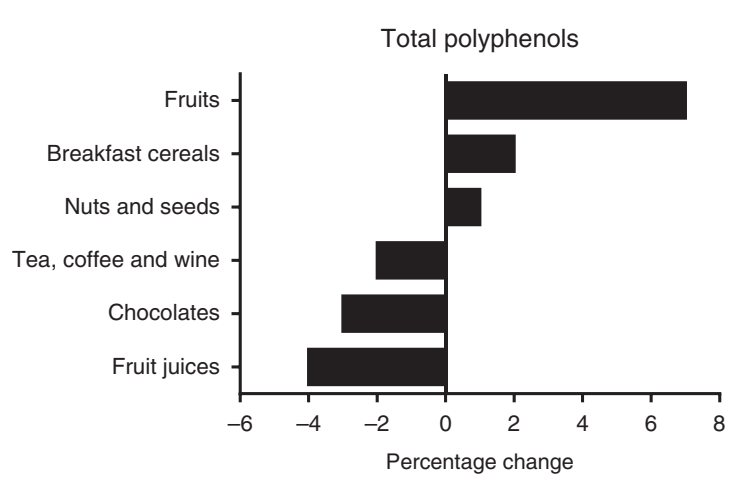

Flavanols
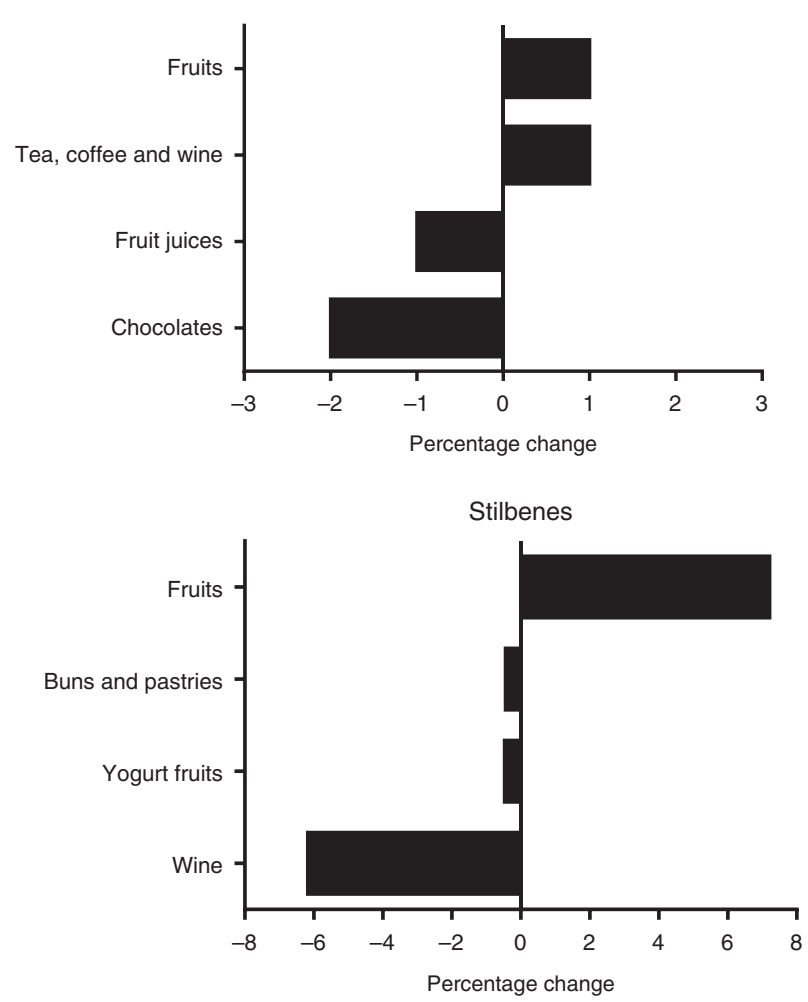
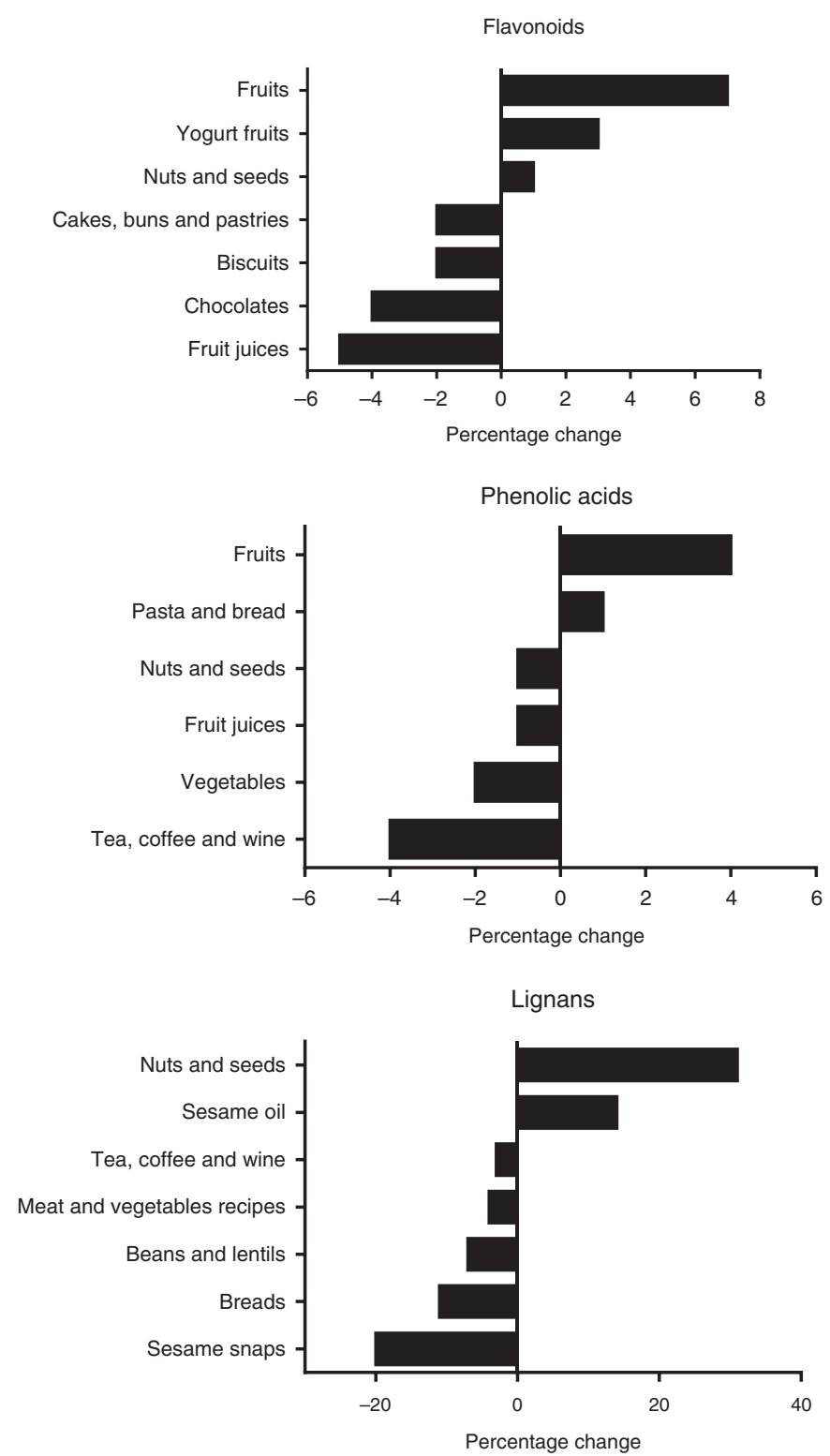

Fig. 3. Percentage changes from baseline in main food group sources of polyphenols in dietary guidelines group as estimated by a 4-d food diary.

Table 3. Daily intake of flavonoids estimated by FFQ in dietary guidelines and control groups* (Medians and interquartile ranges (IQR))

\begin{tabular}{|c|c|c|c|c|c|c|c|}
\hline \multirow{3}{*}{$\begin{array}{l}\text { Flavonoids }(\mathrm{mg} / \mathrm{d}) \\
\text { Class and subclasses }\end{array}$} & & & \multicolumn{4}{|c|}{ Endpoint ( $n$ 161) } & \multirow{3}{*}{$\begin{array}{l}\text { Differences between } \\
\text { groups at endpoint }(P \dagger)\end{array}$} \\
\hline & \multicolumn{2}{|c|}{ Baseline ( $n$ 161) } & \multicolumn{2}{|c|}{ Control ( $n$ 82) } & \multicolumn{2}{|c|}{ Dietary guidelines ( $n$ 79) } & \\
\hline & Median & IQR & Median & IQR & Median & IQR & \\
\hline Total flavonoids & 661 & 417,867 & 539 & 350,862 & 715 & 571,906 & 0.005 \\
\hline Anthocyanins & 19 & 10,35 & 16 & 9,31 & 32 & 19,50 & $<0.001$ \\
\hline Flavones & $2 \cdot 4$ & $1.6,3.5$ & $2 \cdot 7$ & $1.5,3.7$ & $3 \cdot 2$ & $2 \cdot 0,4 \cdot 4$ & 0.013 \\
\hline Flavonols & 35 & 23,47 & 39 & 21,54 & 39 & 31,50 & 0.199 \\
\hline Flavanols & 90 & 40,143 & 85 & 35,146 & 97 & 80,143 & 0.048 \\
\hline Flavanones & 23 & 8,42 & 25 & 10,43 & 27 & 8,49 & 0.352 \\
\hline Proanthocyanidins & 265 & 184,379 & 232 & 184,371 & 290 & 229,385 & 0.021 \\
\hline
\end{tabular}

* There were no significant differences between groups at baseline.

$\dagger$ Statistical comparisons between groups at endpoint by Mann-Whitney $U$ test. 
Table 4. Comparison of urinary phenolic excretion method and polyphenol intake estimated by a 4-d food diary in their ability to discriminate between subsample populations adhering to either dietary guidelines or a control diet†‡

(Medians and interquartile ranges (IQR))

\begin{tabular}{|c|c|c|c|c|c|c|c|c|c|c|}
\hline \multirow[b]{3}{*}{ Polyphenols } & \multicolumn{5}{|c|}{ Urinary excretion§ $(\mu \mathrm{mol} / \mathrm{d})$} & \multicolumn{5}{|c|}{ Dietary intake (mg/d per $10 \mathrm{MJ})$} \\
\hline & \multicolumn{2}{|c|}{ Control ( $n$ 46) } & \multicolumn{2}{|c|}{$\begin{array}{l}\text { Dietary guidelines } \\
\qquad(n \text { 45) }\end{array}$} & \multirow{2}{*}{$\begin{array}{l}\text { Difference between } \\
\text { groups }(P)\end{array}$} & \multicolumn{2}{|c|}{ Control ( $n$ 46) } & \multicolumn{2}{|c|}{$\begin{array}{l}\text { Dietary guidelines } \\
\qquad(n \text { 45) }\end{array}$} & \multirow{2}{*}{$\begin{array}{l}\text { Difference between } \\
\text { groups }(P)\end{array}$} \\
\hline & Median & IQR & Median & IQR & & Median & IQR & Median & IQR & \\
\hline Phloretinll & 0.09 & $0.01,0.28$ & $0.40^{*}$ & $0.18,0.97$ & $<0.001$ & $1 \cdot 2$ & $0.0,3.5$ & $3 \cdot 1^{*}$ & $1 \cdot 1,5.9$ & 0.006 \\
\hline Epicatechin & 0.45 & $0.24,0.60$ & 0.44 & $0.21,0.70$ & 0.943 & 30 & 15,50 & $40^{*}$ & 29,67 & 0.013 \\
\hline Eriodictyol & 0.25 & $0.09,0.43$ & $0.51^{*}$ & $0.20,0.86$ & 0.011 & 0.00 & $0.00,0.02$ & $0.02^{*}$ & $0.01,0.06$ & $<0.001$ \\
\hline Hesperetin & 0.20 & $0.00,2.03$ & $1.38^{*}$ & $0.09,3.51$ & 0.010 & 8 & 1,23 & $17^{*}$ & 4,35 & 0.019 \\
\hline Luteolin & 1.8 & $0.9,2.9$ & $2 \cdot 7^{\star}$ & $1 \cdot 5,3 \cdot 7$ & 0.017 & 0.5 & $0 \cdot 3,-8$ & $1.5^{\star}$ & $0.9,3.3$ & $<0.001$ \\
\hline Quercetin & 4.7 & $1.5,7.4$ & $6 \cdot 6$ & $2 \cdot 4,12 \cdot 8$ & 0.105 & 50 & 27,66 & $59^{*}$ & 41,85 & 0.028 \\
\hline Daidzein & 0.02 & $0.00,0.40$ & 0.11 & $0.00,0.80$ & 0.074 & 0.04 & $0.04,0.10$ & $3 \cdot 5^{\star}$ & $0.02,21.2$ & 0.006 \\
\hline Gallic acid & 0.7 & $0 \cdot 2,2 \cdot 0$ & 1.4 & $0.5,3.6$ & 0.053 & 62 & 18,107 & 90 & 23,153 & 0.134 \\
\hline Vanillic acid & 1.4 & $0.2,2.6$ & $3 \cdot 0^{*}$ & $0.9,5.9$ & 0.023 & 0.2 & $0.1,0.2$ & $0.4^{*}$ & $0.2,0.6$ & $<0.001$ \\
\hline Lignan/enterolactoneף & $5 \cdot 7$ & $2 \cdot 1,10 \cdot 6$ & $10 \cdot 1^{\star}$ & $3 \cdot 8,22 \cdot 6$ & 0.010 & 0.2 & $0.1,0.4$ & $0 \cdot 6^{*}$ & $0.3,6 \cdot 1$ & $<0.001$ \\
\hline
\end{tabular}

* Statistically significant differences between groups at endpoint $(P<0.05)$.

† Excretion of polyphenols in $24 \mathrm{~h}$ urine and dietary intake of the direct dietary precursor estimated by a 4-d food diaries at endpoint in a subsample of the Cardiovascular risk REduction Study: Supported by an Integrated Dietary Approach study population selected for the lowest (control group) and highest (dietary guidelines group) fruit and vegetables intake using self-reported data from FFQ $(n$ 91).

$\ddagger$ All between-group comparisons were carried out by independent-samples Mann-Whitney $U$ test.

$\S$ Values adjusted for $24 \mathrm{~h}$ urine volume.

II Sum of phloretin and phlorizin.

If Intake of lignans was used as a direct dietary precursor of enterolactone.

eriodictyol, hesperetin and syringic acid), nuts and seeds (secoisolariciresinol and matairesinol) and soya products (daidzein, genistein and other isoflavonoids) compared with the control group. In agreement with the 4-DFD results, FFQ estimates of total flavonoids, proanthocyanidins and anthocyanins were higher in the DG group compared with the control group. A total of ten representative aglycone polyphenols and phenolic acids (from glucuronidated and sulphated metabolites) were measured in $24 \mathrm{~h}$ urine samples collected concurrently with 4-DFD as the objective biomarkers of (poly)phenol intake, to determine the accuracy of the dietary intake estimates. The ability to discriminate between the dietary group subsamples was consistent with 4-DFD, except for epicatechin, daidzein and quercetin where there were no significant differences in urinary excretion in contrast with higher estimated intake in the DG group by 4-DFD. Both the 4-DFD and urinary biomarker methods agreed that intake of phlorizin/phloretin, eriodictyol, hesperidin, luteolin, vanillic acid and lignans/enterolactone was greater in the DG group.

The ability to discriminate between dietary intervention groups supports the utility of 4-DFD and urinary metabolites for assessment of dihydrochalcones, flavanones, flavones, hydroxybenzoic acids and lignan intake over short-term periods. Differing findings may be due to the incomplete phenolic composition data, the well-known inaccuracies of self-reported dietary assessment methodology ${ }^{(68,69)}$ and the inter-individual variability in absorption and metabolism ${ }^{(8,70)}$ or incomplete deconjugation of sulphated/glucuronidated metabolites ${ }^{(71)}$ reflected in the urinary biomarkers. Urine polyphenol concentrations may also be the sum product of endogenous metabolism and dietary intake. For example, gallic acid is a common metabolite of black tea theaflavins and thearubigins $^{(72,73)}$; therefore, its urinary excretion could reflect the consumption of such beverage combined with foods providing gallic acid per se. Vanillic acid can be found in high concentrations in foods consumed in small quantities such as herbs; also in moderate concentration in dates, olives and cranberries and in low concentrations in foods more frequently consumed like oats and rice ${ }^{(39)}$. It is also a common metabolite of anthocyanins ${ }^{(74,75)}$, and variability in urinary vanillic acid excretion could reflect varying intake of both vanillic acid and anthocyanin-rich foods, therefore it is likely that the increased urinary excretion at endpoint mainly represents intake of foods rich in anthocyanins rather than vanillic acid. Vanillic acid is also reported to be present in urine after consumption of black tea ${ }^{(76)}$ and dark chocolate ${ }^{(77)}$; however, intakes of black tea and dark chocolate were not higher in the DG group at endpoint and, therefore, are unlikely to account for the increased urinary excretion and reported intake in the 4-DFD. Enterolactone is the main colonic metabolite of lignans, which are phytoestrogenic compounds present in high concentrations in vegetables, cereals and grain products, seeds, nuts and berries and other fruits ${ }^{(65,78)}$. These urinary excretion data are in agreement with previous epidemiologial studies ${ }^{(79)}$, supplementation studies where known doses of polyphenols were administrated ${ }^{(44,80)}$, randomised controlled trial (RCT) aiming to increase flavonid-rich $\mathrm{F} \& \mathrm{~V}$ intake ${ }^{(81)}$ and population under free-living conditions ${ }^{(43)}$. Polyphenol urinary excretion has shown to be an useful biomarker of F\&V intake, capable of detecting even small changes in studies under controlled diets ${ }^{(45,82,83)}$.

Following DG did lead to increase in specific polyphenols that have been associated with health benefits (e.g. anthocyanins, flavanones, isoflavones, phenolic acids and lignans ${ }^{(84-87)}$ ). This suggests that consuming at least 5 portions of $F \& V / d$ and $>50 \%$ of cereal intake as wholegrain will lead to an enrichment in dietary polyphenol profiles above and beyond the large amounts of polyphenols supplied by beverages. In particular, estimated anthocyanidin intake was augmented by following 
DG that may confer a reduction in the risk of $\mathrm{T} 2 \mathrm{D}^{(25)}$ and acute reductions in postprandial glycaemia ${ }^{(88-91)}$. The dietary intake results reported here are consistent with the theory that greater intake of specific polyphenols may contribute to the protective effects of a diet rich in fruits and vegetables and wholegrain cereals, alongside greater dietary intake of nutrients and other non-nutrient bioactives, for example, K, vitamin C, soluble fibre, carotenoids and glucosinolates. However, our results confirm ${ }^{(29-31)}$ that food groups that are recommended by UK government as part of the Eatwell Guide - fruits and vegetables, wholegrain cereals, beans, pulses, nuts and seeds - contribute a much smaller proportion of daily polyphenol intake in a UK population compared with commonly consumed beverages, even when that population is following DG. Analysis of dietary data showed no group differences in (poly)phenol intake (by 4-DFD) where main food sources were not targets for UK DG such as tea (catechins, theaflavins, proanthocyanidins, quercetin) and wine (stilbenes, proanthocyanidins). However, the sources of these polyphenols may have differed between groups; for example, the DG group reported lower intake of stilbenes from wine and increased intake of stilbenes from fruits.

The relative impact of total and specific (poly)phenol intake on risk of CVD and type 2 diabetes requires investigation. Clearly there is only limited scope for increasing total polyphenol intake by following DG - an approximate increase of $200 \mathrm{mg} / \mathrm{d}$ per $10 \mathrm{MJ}$ energy intake. The extent to which this is relevant to cardiometabolic health is currently unclear due to the lack of robust evidence for the relative impact of different polyphenol subclasses on risk factors for CVD and type 2 diabetes. Although the evidence is currently insufficient, it is plausible to hypothesise that in the future there may be consensus that tea and coffee (poly)phenols ${ }^{(92,93)}$ may have some of the strongest cardiometabolic protective effects amongst all dietary phenolics, so that due to the large amounts of tea and coffee consumed by a population level, the gain to health in consuming at least 5 portions/d of fruits and vegetables and choosing wholegrain sources of starchy carbohydrates is likely to be due to other nutritional factors. Conversely, future advances in nutritional science may $1 \mathrm{~d}$ demonstrate that certain polyphenols specific to fruits, vegetables or wholegrain cereals have particularly potent bioactivity in preventing inflammation, atherosclerosis or insulin resistance, which would bring about a departure from the prevailing approach of considering all plant chemicals that contain a similar chemical structure in the same way. There has been some debate around whether establishing a dietary reference intake for polyphenols could be beneficial; with suggested approaches including recommendations for polyphenol-rich F\&V (5-a-day) ${ }^{(52)}$, establishing a specific daily dose for a given effect ${ }^{(54)}$ or establishing values to improve health or prevent disease risk in different life stages ${ }^{(53)}$. Further research is required to provide robust interventional evidence for any refinement to current '5-a-day' guidelines, since the relative health impact of a multitude of other non-nutrient bioactives and the complex array of plant cell wall polysaccharides, resistant starch and oligosaccharides (collectively known as fibre) contained in low-polyphenol plant-based foods is only partially understood. Furthermore, in the light of the fact that NDNS survey data suggest that the UK population are not meeting current recommendations for $F \& V$ then it would be futile, and possibly counter-productive, to add further complexity to existing public health dietary advice.

The validation of adequate biomarkers of dietary intake of exposure to dietary components and of compliance to dietary interventions has been a focus of investigation for many years $^{(94)}$, and the identification of polyphenol intake biomarkers is no exception ${ }^{(83,95)}$. Assessment of compliance in dietary intervention studies with fruits and vegetables has been done by validated biomarkers ${ }^{(96)}$ such as vitamin C, carotenoids and $\mathrm{K}$, however, polyphenol excretion in urine may be used to assess flavonoid-rich dietary interventions ${ }^{(81)}$ or specific $\mathrm{F} \& \mathrm{~V}$ intake, for example, phloretin for apple intake and naringenin and hesperetin for citrus fruit ${ }^{(95,97)}$. Given the importance of accurate assessment of polyphenol intake to link it with beneficial effects on health ${ }^{(98)}$, the ability to detect the amount consumed represents a challenge in epidemiological and clinical studies.

The bespoke food composition table developed for the 4-DFD polyphenol analysis in the present study included the estimation of fifty-two individual compounds and contained 1141 foods representative of an average UK diet as well as some of the most commonly eaten food from non-traditional UK cuisine as Turkish, Indian, Italian, Chinese, Japanese and others. This food composition table could be used as a basis for future studies analysing diet patterns in the UK population, comparing polyphenols intake and/or linking intake with health benefits, although it would require a significant staff cost commitment to maintain and update with new recipes and new versions of Phenol-Explorer over time. Although an onerous process, the creation of a bespoke food composition table for polyphenol analysis in a specific population seems to be the most effective way to obtain an accurate estimation of polyphenol intake. Although the FFQ is a valid and reliable dietary assessment tool for estimation of habitual intake in populations, it has limitations in the way that it can collect information, since it is based on uniform estimated portion sizes and is semi-quantitative. Dietary intake of total flavonoid intake, six flavonoid subclasses and thirty-two individual flavonoids (aglycones) were estimated by FFQ (EPIC-Norfolk) using the methodology previously applied in large cohort studies ${ }^{(15,17,23,25,26,99)}$. The EPIC-Norfolk FFQ, although validated for whole foods intake ${ }^{(100)}$, has not been validated for flavonoid intake. Methodological barriers of this approach in regard to flavonoid estimation are particularly limiting in smaller study cohorts: some foods are grouped incongruously with regard to their flavonoid composition such as 'strawberries, raspberries and kiwi', or 'peaches, plums and apricots' and 'wine' (no separation of red, white and rose). Some important flavonoid sources are missing such as blueberries, soya beverages, lemon, among others. The inadequacy of this method could lead to under- or overestimation of flavonoid intake. Studies have used food diaries for estimation of flavanol intake among European population ${ }^{(29)}$, isoflavone and lignan intake ${ }^{(66)}$ and twenty subclasses of polyphenols ${ }^{(31)}$ among the UK population, each one creating their own food composition table for polyphenol analysis; however, they have not included objective biomarkers for corroborating agreement 
between methods. More specialised questionnaires for intake estimation have been developed for individual compounds like quercetin and naringenin ${ }^{(101)}$, flavonoid subclasses such as flavonols and flavones among a Chinese population ${ }^{(102)}$, complete flavonoid class among Australian population ${ }^{(34)}$ and Flemish population $^{(103)}$. In summary, although the FFQ results reported here were in broad agreement with the 4-DFD, regarding the discernment of differences in total flavonoid intake in a dietary intervention study, future research in this area could include the development of a specific FFQ, validated among the UK population, for estimation of phenolic intake including all classes and main subclasses as were estimated by 4-DFD in this study.

Limitations in the present study include the hydrolysis of urinary polyphenols by glucuronidase/sulphatase enzymes before analysis, which could compromise the stability of polyphenols that are to be quantified ${ }^{(104,105)}$. Glucuronidase/ sulphatase enzymes may fail to completely hydrolyse all sulphated and methylated-sulphated metabolites, which may have been compounded by the addition of boric acid as a preservative to prevent yeast growth, and, therefore, urinary excretion of the corresponding aglycone could be underestimated $^{(71)}$. Furthermore, our approach required preselection of polyphenols to be analysed, which means that many other polyphenols present in $24 \mathrm{~h}$ urine that may have also functioned as biomarkers of intake were not detected and quantified. Although the reported anthocyanidin intake was greater in the DG group, we did not attempt to analyse anthocyanins in urine as they have low recovery as parent compounds and only weak correlations with the dose ingested, necessitating the use of stable isotopes to accurately measure the excretion of anthocyanin metabolites ${ }^{(44)}$. The 4 -DFD proved to be an accurate approach for estimating short-term polyphenol intake, however, serious consideration must be made of the potential researcher burden and cost if using these methods in largerscale studies, particularly where a simple indicator of compliance to dietary advice is required.

Strengths of our study include the fact that the data collected in this study enabled a comparison of two dietary assessment methods with objective biomarkers of (poly)phenol dietary intake collected concurrently towards the end of the dietary intervention period. To date, this three-way comparison has only been reported previously in a cross-sectional ${ }^{(106)}$ study using a crude estimate of total urinary polyphenol excretion by the Folin-Ciocalteu assay. To the best of our knowledge, this is the first study to use the three way comparison on data obtained from an RCT allowing comparison of change from baseline for individuals advised to consume a diet consistent with DG.

In conclusion, participants following advice to adhere the UK DG consumed a greater amount of total polyphenols than a control group consuming a representative UK diet. In particular, intake of individual polyphenols that are mainly sourced from fruits and vegetables, nuts, seeds and soya products was increased in the DG group. The results of this study advance the field of polyphenol research as they demonstrate that a diet consistent with DG is also moderately richer in polyphenols compared with a typical UK dietary pattern, despite the relatively high baseline intake derived from commonly consumed beverages such as tea and coffee. In addition to increased intake of dietary fibre and certain micronutrients, replacement of SFA with unsaturated fatty acids, and lower salt and free sugars, following DG will also increase polyphenol intake, which may contribute to the overall reduction in risk factors for cardiometabolic diseases.

\section{Acknowledgements}

The authors thank Anna Caldwell (CEMS-Waterloo, King's College London) for her assistance with sample analysis, and Ana Rodriguez-Mateos (Department of Nutritional Sciences, King's College London) for reading and commenting on the initial manuscript draft.

The CRESSIDA study received funding from the UK Food Standards Agency and Department of Health and from the National Institute for Health Research (NIHR) Clinical Research Facility at Guy's and St Thomas' NHS Foundation Trust and NIHR Biomedical Research Centre based at Guy's and St Thomas' NHS Foundation Trust and King's College London. M. L. C.-A. received $\mathrm{PhD}$ studentship funding from the Mexican Secretariat of Public Education.

T. A. B. S. was principal investigator of the CRESSIDA study. T. A. B. S. and W. L. H. devised the CRESSIDA study, D. P. R. and J. D. recruited subjects into the study and supported the dietary intervention in the CRESSIDA study. M. L. C.-A. and W. L. H. conceived the idea for secondary analysis of polyphenols intake data, analysed data and performed statistical analysis. M. L. C.-A. wrote drafts of the manuscripts, which were edited by W. L. H. The manuscript was read, commented on and approved by all authors.

W. L. H. has collaborated with Lucozade Ribena Suntory and DIANA Food SAS. T. A. B. S., D. P. R., J. D. and M. L. C.-A. reported no conflicts of interest.

\section{References}

1. Boeing H, Bechthold A, Bub A, et al. (2012) Critical review: vegetables and fruit in the prevention of chronic diseases. Eur J Nutr 51, 637-663.

2. Muraki I, Imamura F, Manson JE, et al. (2013) Fruit consumption and risk of type 2 diabetes: results from three prospective longitudinal cohort studies. BMJ 347, f5001.

3. Mursu J, Virtanen JK, Tuomainen TP, et al. (2014) Intake of fruit, berries, and vegetables and risk of type 2 diabetes in Finnish men: the Kuopio Ischaemic Heart Disease Risk Factor Study. Am J Clin Nutr 99, 328-333.

4. Buil-Cosiales P, Toledo E, Salas-Salvado J, et al. (2016) Association between dietary fibre intake and fruit, vegetable or whole-grain consumption and the risk of CVD: results from the PREvencion con DIeta MEDiterranea (PREDIMED) trial. Br J Nutr 116, 534-546.

5. Aune D, Keum N, Giovannucci E, et al. (2016) Whole grain consumption and risk of cardiovascular disease, cancer, and all cause and cause specific mortality: systematic review and dose-response meta-analysis of prospective studies. BMJ 353, i2716.

6. Ötles S \& Ozgoz S (2014) Health effects of dietary fiber. Acta Sci Pol Technol Aliment 12, 191-202. 
7. Woodside JV, McCall D, McGartland C, et al. (2005) Micronutrients: dietary intake $v$. supplement use. Proc Nutr Soc 64, 543-553.

8. Rodriguez-Mateos A, Vauzour D, Krueger CG, et al. (2014) Bioavailability, bioactivity and impact on health of dietary flavonoids and related compounds: an update. Arch Toxicol 88, 1803-1853.

9. World Health Organization (1990) Diet, Nutrition and the Prevention of Chronic Diseases. Technical Report Series 797. Geneva: World Health Organization.

10. NHS (2005) 5 a day - live well. http://www.nhs.uk/livewell/ 5aday/Pages/5ADAYhome.aspx (accessed 16 March 2016).

11. Bates B, Lennox A, Prentice A, et al. (2014) National Diet and Nutrition Survey: Results from years 1-4 (Combined) of the Rolling Programme (2008/2009-2011/12) Executive Summary. London: Public Health England.

12. Mann KD, Pearce MS, McKevith B, et al. (2015) Low whole grain intake in the UK: results from the National Diet and Nutrition Survey rolling programme 2008-11. Br J Nutr $\mathbf{1 1 3}$, 1643-1651.

13. Manach C, Scalbert A, Morand C, et al. (2004) Polyphenols: food sources and bioavailability. Am J Clin Nutr 79, $727-747$

14. Hooper L, Kroon PA, Rimm EB, et al. (2008) Flavonoids, flavonoid-rich foods, and cardiovascular risk: a metaanalysis of randomized controlled trials. Am J Clin Nutr $\mathbf{8 8}, 38-50$

15. Jennings A, Welch AA, Fairweather-Tait SJ, et al. (2012) Higher anthocyanin intake is associated with lower arterial stiffness and central blood pressure in women. Am J Clin Nutr 96, 781-788.

16. McCullough ML, Peterson JJ, Patel R, et al. (2012) Flavonoid intake and cardiovascular disease mortality in a prospective cohort of US adults. Am J Clin Nutr 95, 454-464.

17. Cassidy A, Mukamal KJ, Liu L, et al. (2013) High anthocyanin intake is associated with a reduced risk of myocardial infarction in young and middle-aged women. Circulation 127, 188-196.

18. van Dam RM, Naidoo N \& Landberg R (2013) Dietary flavonoids and the development of type 2 diabetes and cardiovascular diseases: review of recent findings. Curr Opin Lipidol 24, 25-33.

19. Neuhouser ML (2004) Dietary flavonoids and cancer risk: evidence from human population studies. Nutr Cancer 50, $1-7$.

20. Arts IC \& Hollman PC (2005) Polyphenols and disease risk in epidemiologic studies. Am J Clin Nutr 81, 317S-325S.

21. Fink BN, Steck SE, Wolff MS, et al. (2007) Dietary flavonoid intake and breast cancer survival among women on Long Island. Cancer Epidemiol Biomarkers Prev 16, 2285-2292.

22. Yuan JM (2011) Green tea and prevention of esophageal and lung cancers. Mol Nutr Food Res 55, 886-904

23. Wedick NM, Pan A, Cassidy A, et al. (2012) Dietary flavonoid intakes and risk of type 2 diabetes in US men and women. Am J Clin Nutr 95, 925-933.

24. Curtis PJ, Sampson M, Potter J, et al. (2012) Chronic ingestion of flavan-3-ols and isoflavones improves insulin sensitivity and lipoprotein status and attenuates estimated 10-year CVD risk in medicated postmenopausal women with type 2 diabetes: a 1-year, double-blind, randomized, controlled trial. Diabetes Care 35, 226-232.

25. Jennings A, Welch AA, Spector T, et al. (2014) Intakes of anthocyanins and flavones are associated with biomarkers of insulin resistance and inflammation in women. J Nutr 144, 202-208.
26. Jacques PF, Cassidy A, Rogers G, et al. (2013) Higher dietary flavonol intake is associated with lower incidence of type 2 diabetes. J Nutr 143, 1474-1480.

27. Zamora-Ros R, Knaze V, Lujan-Barroso L, et al. (2011) Estimated dietary intakes of flavonols, flavanones and flavones in the European Prospective Investigation into Cancer and Nutrition (EPIC) 24 hour dietary recall cohort. Br J Nutr 106, $1915-1925$

28. Zamora-Ros R, Knaze V, Lujan-Barroso L, et al. (2011) Estimation of the intake of anthocyanidins and their food sources in the European Prospective Investigation into Cancer and Nutrition (EPIC) study. Br J Nutr 106, 1090-1099.

29. Vogiatzoglou A, Mulligan AA, Luben RN, et al. (2013) Assessment of the dietary intake of total flavan-3-ols, monomeric flavan-3-ols, proanthocyanidins and theaflavins in the European Union. Br J Nutr 111, 1463-1473.

30. Zamora-Ros R, Knaze V, Rothwell JA, et al. (2015) Dietary polyphenol intake in Europe: the European Prospective Investigation into Cancer and Nutrition (EPIC) study. Eur J Nutr 55, 1359-1375.

31. Yahya HM, Day A, Lawton C, et al. (2015) Dietary intake of 20 polyphenol subclasses in a cohort of UK women. Eur J Nutr 55, 1839-1847.

32. Pinto P \& Santos CN (2017) Worldwide (poly)phenol intake: assessment methods and identified gaps. Eur J Nutr 56, 1393-1408.

33. Naska A, Lagiou A \& Lagiou P (2017) Dietary assessment methods in epidemiological research: current state of the art and future prospects. F1000Res 6, 926.

34. Somerset S \& Papier K (2014) A food frequency questionnaire validated for estimating dietary flavonoid intake in an Australian population. Nutr Cancer 66, 1200-1210.

35. Theodoratou E, Kyle J, Cetnarskyj R, et al. (2007) Dietary flavonoids and the risk of colorectal cancer. Cancer Epidemiol Biomarkers Prev 16, 684-693.

36. Zamora-Ros R, Touillaud M, Rothwell JA, et al. (2014) Measuring exposure to the polyphenol metabolome in observational epidemiologic studies: current tools and applications and their limits. Am J Clin Nutr 100, 11-26.

37. Neveu V, Perez-Jimenez J, Vos F, et al. (2010) PhenolExplorer: an online comprehensive database on polyphenol contents in foods. Database (Oxford) 2010, bap024.

38. Rothwell JA, Urpi-Sarda M, Boto-Ordonez M, et al. (2012) Phenol-Explorer 2.0: a major update of the Phenol-Explorer database integrating data on polyphenol metabolism and pharmacokinetics in humans and experimental animals. Database (Oxford) 2012, bas031.

39. Rothwell JA, Perez-Jimenez J, Neveu V, et al. (2013) PhenolExplorer 3.0: a major update of the Phenol-Explorer database to incorporate data on the effects of food processing on polyphenol content. Database 2013, bat070.

40. US Department of Agriculture, Agricultural Research Service (2004) Database for the Proanthocyanidin Content of Selected Foods. Nutrient Data Laboratory Home Page. http://www.ars.usda.gov/nutrientdata/flav

41. Bhagwat S, Haytowitz DB \& Holden JM (2008) USDA Database for the Isoflavone Content of Selected Foods, Release 2.0. U.S. Department of Agriculture, Agricultural Research Service. Nutrient Data Laboratory Home Page. http://www.ars.usda.gov/nutrientdata/isoflav

42. Bhagwat S, Haytowitz DB \& Holden (Ret.) JM (2013) USDA Database for the Flavonoid Content of Selected Foods, Release 3.1. U.S. Department of Agriculture, Agricultural Research Service. Nutrient Data Laboratory Home Page. http://www.ars.usda.gov/nutrientdata/flav 
43. Mennen LI, Sapinho D, Ito H, et al. (2008) Urinary excretion of 13 dietary flavonoids and phenolic acids in free-living healthy subjects - variability and possible use as biomarkers of polyphenol intake. Eur J Clin Nutr 62, 519-525.

44. Perez-Jimenez J, Hubert J, Hooper L, et al. (2010) Urinary metabolites as biomarkers of polyphenol intake in humans: a systematic review. Am J Clin Nutr 92, 801-809.

45. Nielsen S, Freese R, Kleemola P, et al. (2002) Flavonoids in human urine as biomarker for intake of fruits and vegetables. Cancer Epidemiol Biomarkers Prev 11, 459-466.

46. Krogholm KS, Haraldsdo J, Knuthsen P, et al. (2004) Urinary total flavonoid excretion but not 4-pyridoxic acid or potassium can be used as a biomarker for the intake of fruits and vegetables. J Nutr 134, 445-451.

47. Ito H, Gonthier M-P, Manach C, et al. (2005) Polyphenol levels in human urine after intake of six different polyphenol-rich beverages. Br J Nutr 94, 500-509.

48. Mullen W, Borges G, Lean ME, et al. (2010) Identification of metabolites in human plasma and urine after consumption of a polyphenol-rich juice drink. J Agric Food Chem 58, 2586-2595.

49. Mennen LI, Sapinho D, Ito H, et al. (2006) Urinary flavonoids and phenolic acids as biomarkers of intake for polyphenolrich foods. BrJ Nutr 96, 191-198.

50. Edmands WM, Ferrari P, Rothwell JA, et al. (2015) Polyphenol metabolome in human urine and its association with intake of polyphenol-rich foods across European countries. Am J Clin Nutr 102, 905-913.

51. Spencer JP, Abd El Mohsen MM, Minihane AM, et al. (2008) Biomarkers of the intake of dietary polyphenols: strengths, limitations and application in nutrition research. Br J Nutr 99, 12-22.

52. Williamson G \& Holst B (2008) Dietary reference intake (DRI) value for dietary polyphenols: are we heading in the right direction? Br J Nutr 99, Suppl. 3, S55-S58.

53. Lupton JR, Atkinson SA, Chang N, et al. (2014) Exploring the benefits and challenges of establishing a DRI-like process for bioactives. Eur J Nutr 53, Suppl. 1, 1-9.

54. Williamson G (2017) The role of polyphenols in modern nutrition. Nutr Bull 42, 226-235.

55. Perez-Jimenez J, Neveu V, Vos F, et al. (2010) Systematic analysis of the content of 502 polyphenols in 452 foods and beverages: an application of the Phenol-Explorer database. J Agric Food Chem 58, 4959-4969.

56. Reidlinger DP, Darzi J, Hall WL, et al. (2015) How effective are current dietary guidelines for cardiovascular disease prevention in healthy middle-aged and older men and women? A randomized controlled trial. Am J Clin Nutr 101, 922-930.

57. Reidlinger DP (2015) Evidence, benefits and barriers to achieving an integrated cardioprotective dietary pattern. PhD Thesis, King's College London.

58. Finglas PM, Roe MA, Pinchen HM, et al. (2015) McCance and Widdowson's The Composition of Foods, 7th Summary ed. Cambridge: Royal Society of Chemistry.

59. Campbell J, Foskett D, Rippington N, et al. (2012) Practical Cookery, 12th ed. London: Hodder Education.

60. BBC Worldwide (2009) BBC good food. http://www. bbcgoodfood.com/ (accessed August 2013).

61. Bingham SA, Gill C, Welch A, et al. (1994) Comparison of dietary assessment methods in nutritional epidemiology: weighed records $v$. $24 \mathrm{~h}$ recalls, food frequency questionnaires and estimated diet records. BrJ Nutr 72, 619-642.

62. Achaintre D, Buleté A, Cren-Olivé C, et al. (2016) Differential isotope labeling of 38 dietary polyphenols and their quantification in urine by liquid chromatography electrospray ionization tandem mass spectrometry. Anal Chem $\mathbf{8 8}$, 2637-2644

63. Bingham S \& Cummings JH (1983) The use of 4-aminobenzoic acid as a marker to validate the completeness of $24 \mathrm{~h}$ urine collections in man. Clin Sci 64, 629-635.

64. Rhee JJ, Sampson L, Cho E, et al. (2014) Comparison of methods to account for implausible reporting of energy intake in epidemiologic studies. Am J Epidemiol 181, 225-233.

65. Tetens I, Turrini A, Tapanainen H, et al (2013) Dietary intake and main sources of plant lignans in five European countries. Food Nutr Res 57, 10.3402/fnr.v57i0.19805.

66. Mulligan AA, Kuhnle GG, Lentjes MA, et al. (2013) Intakes and sources of isoflavones, lignans, enterolignans, coumestrol and soya-containing foods in the Norfolk arm of the European Prospective Investigation into Cancer and Nutrition (EPIC-Norfolk), from $7 \mathrm{~d}$ food diaries, using a newly updated database. Public Health Nutr 16, 1454-1462.

67. Knaze V, Zamora-Ros R, Lujan-Barroso L, et al. (2012) Intake estimation of total and individual flavan-3-ols, proanthocyanidins and theaflavins, their food sources and determinants in the European Prospective Investigation into Cancer and Nutrition (EPIC) study. Br J Nutr 108, 1095-1108.

68. Bingham SA, Gill C, Welch A, et al. (1994) Comparison of dietary assessment methods in nutritional epidemiology: weighed records $v$. $24 \mathrm{~h}$ recalls, food frequency questionnaires and estimated diet records. Br J Nutr 72, 619-642.

69. Zamora-Ros R, Rabassa M, Llorach R, et al. (2012) Application of dietary phenolic biomarkers in epidemiology: past, present, and future. J Agric Food Chem 60, 6648-6657.

70. Chen Z, Zheng S, Li L, et al. (2014) Metabolism of flavonoids in human: a comprenhensive review. Curr Drug Metab 15, 48-61.

71. Saha S, Hollands W, Needs PW, et al. (2012) Human O-sulfated metabolites of (-)-epicatechin and methyl(-)-epicatechin are poor substrates for commercial aryl-sulfatases: implications for studies concerned with quantifying epicatechin bioavailability. Pharmacol Res 65, 592-602.

72. Henning SM, Wang P, Abgaryan N, et al. (2013) Phenolic acid concentrations in plasma and urine from men consuming green or black tea and potential chemopreventive properties for colon cancer. Mol Nutr Food Res 57, 483-493.

73. Crozier A, Clifford MN \& Del rio D (2012) Bioavalability of dietary monomeric and polymeric flavan-3-ols. In Flavonoids and Related Compounds, pp. 45-78 [JPE Spencer and A Crozier, editors]. Boca Raton, FL: CRC Press.

74. Nurmi T, Mursu J, Heinonen M, et al. (2009) Metabolism of berry anthocyanins to phenolic acids in humans. J Agric Food Chem 57, 2274-2281.

75. de Ferrars RM, Cassidy A, Curtis P, et al. (2013) Phenolic metabolites of anthocyanins following a dietary intervention study in post-menopausal women. Mol Nutr Food Res 58, 490-502.

76. Olthof MR, Hollman PC, Buijsman MN, et al. (2003) Chlorogenic acid, quercetin-3-rutinoside and black tea phenols are extensively metabolized in humans. J Nutr $\mathbf{1 3 3}$, 1806-1814.

77. Rios LY, Gonthier MP, Remesy C, et al. (2003) Chocolate intake increases urinary excretion of polyphenol-derived phenolic acids in healthy human subjects. Am J Clin Nutr 77, 912-918.

78. Pounis G, Di Castelnuovo A, Bonaccio M, et al. (2016) Flavonoid and lignan intake in a Mediterranean population: proposal for a holistic approach in polyphenol dietary analysis, the Moli-sani Study. Eur J Clin Nutr 70, 338-345. 
79. Valls-Pedret C, Lamuela-Raventos RM, Medina-Remon A, et al. (2012) Polyphenol-rich foods in the Mediterranean diet are associated with better cognitive function in elderly subjects at high cardiovascular risk. J Alzheimers Dis 29, 773-782.

80. Shi Y \& Williamson G (2015) Comparison of the urinary excretion of quercetin glycosides from red onion and aglycone from dietary supplements in healthy subjects: a randomized, single-blinded, cross-over study. Food Funct $\mathbf{6}$, 1443-1448.

81. Chong MF, George TW, Alimbetov D, et al. (2012) Impact of the quantity and flavonoid content of fruits and vegetables on markers of intake in adults with an increased risk of cardiovascular disease: the FLAVURS trial. Eur J Nutr 52, 361-378.

82. Brevik A, Rasmussen SE, Drevon CA, et al. (2004) Urinary excretion of flavonoids reflects even small changes in the dietary intake of fruits and vegetables. Cancer Epidemiol Biomarkers Prev 13, 843-849.

83. Vetrani C, Rivellese AA, Annuzzi G, et al. (2014) Phenolic metabolites as compliance biomarker for polyphenol intake in a randomized controlled human intervention. Food Res Int 63, 233-238.

84. Garcia-Conesa MT, Chambers K, Combet E, et al. (2018) Meta-analysis of the effects of foods and derived products containing ellagitannins and anthocyanins on cardiometabolic biomarkers: analysis of factors influencing variability of the individual responses. Int J Mol Sci 19, 694.

85. Rienks J, Barbaresko J \& Nothlings U (2017) Association of isoflavone biomarkers with risk of chronic disease and mortality: a systematic review and meta-analysis of observational studies. Nutr Rev 75, 616-641.

86. Onakpoya IJ, Spencer EA, Thompson MJ, et al. (2015) The effect of chlorogenic acid on blood pressure: a systematic review and meta-analysis of randomized clinical trials. J Hum Hypertens 29, 77-81.

87. Testai L \& Calderone V (2017) Nutraceutical value of citrus flavanones and their implications in cardiovascular disease. Nutrients 9, 502 .

88. Törrönen R, Kolehmainen M, Sarkkinen E, et al. (2012) Postprandial glucose, insulin, and free fatty acid responses to sucrose consumed with blackcurrants and lingonberries in healthy women. Am J Clin Nutr 96, 527-533.

89. Törrönen R, Sarkkinen E, Niskanen T, et al. (2012) Postprandial glucose, insulin and glucagon-like peptide 1 responses to sucrose ingested with berries in healthy subjects. Br J Nutr 107, 1445-1451.

90. Törrönen R, Sarkkinen E, Tapola N, et al. (2010) Berries modify the postprandial plasma glucose response to sucrose in healthy subjects. BrJ Nutr 103, 1094-1097.

91. Castro-Acosta ML, Smith L, Miller RJ, et al. (2016) Drinks containing anthocyanin-rich blackcurrant extract decrease postprandial blood glucose, insulin and incretin concentrations. J Nutr Biochem 38, 154-161.
92. Hartley L, Flowers N, Holmes J, et al. (2013) Green and black tea for the primary prevention of cardiovascular disease. Cochrane Database Syst Rev, issue 6, CD009934.

93. Tajik N, Tajik M, Mack I, et al. (2017) The potential effects of chlorogenic acid, the main phenolic components in coffee, on health: a comprehensive review of the literature. Eur J Nutr 56, 2215-2244.

94. Dragsted LO, Gao Q, Pratico G, et al. (2017) Dietary and health biomarkers-time for an update. Genes Nutr 12, 24.

95. Noh H, Freisling H, Assi N, et al. (2017) Identification of urinary polyphenol metabolite patterns associated with polyphenol-rich food intake in adults from four European countries. Nutrients 9, 796.

96. Woodside JV, Draper J, Lloyd A, et al. (2017) Use of biomarkers to assess fruit and vegetable intake. Proc Nutr Soc 76, 308-315.

97. Brouwer-Brolsma EM, Brennan L, Drevon CA, et al. (2017) Combining traditional dietary assessment methods with novel metabolomics techniques: present efforts by the Food Biomarker Alliance. Proc Nutr Soc 76, 619-627.

98. Kuhnle GG (2012) Nutritional biomarkers for objective dietary assessment. J Sci Food Agric 92, 1145-1149.

99. Cassidy A, O'Reilly EJ, Kay C, et al. (2011) Habitual intake of flavonoid subclasses and incident hypertension in adults. Am J Clin Nutr 93, 338-347.

100. McKeown NM, Day NE, Welch AA, et al. (2001) Use of biological markers to validate self-reported dietary intake in a random sample of the European Prospective Investigation into Cancer United Kingdom Norfolk cohort. Am J Clin Nutr 74, 188-196.

101. Ranka S, Gee JM, Biro L, et al. (2008) Development of a food frequency questionnaire for the assessment of quercetin and naringenin intake. Eur J Clin Nutr 62, 1131-1138.

102. Zhang Y, Cao J, Chen W, et al. (2010) Reproducibility and relative validity of a food frequency questionnaire to assess intake of dietary flavonol and flavone in Chinese university campus population. Nutr Res 30, 520-526.

103. Mullie P, Clarys P, Deriemaeker P, et al. (2007) Estimation of daily human intake of food flavonoids. Plant Foods Hum Nutr 62, 93-98.

104. Cheynier V, Tomas-Barberan FA \& Yoshida K (2015) Polyphenols: from plants to a variety of food and nonfood uses. J Agric Food Chem 63, 7589-7594.

105. Ding Y, Peng M, Zhang T, et al. (2013) Quantification of conjugated metabolites of drugs in biological matrices after the hydrolysis with $\beta$-glucuronidase and sufatase: a review of bio-analytical methods. Biomed Chromatogr 27, 1280-1295.

106. Burkholder-Cooley NM, Rajaram SS, Haddad EH, et al. (2017) Validating polyphenol intake estimates from a foodfrequency questionnaire by using repeated 24-h dietary recalls and a unique method-of-triads approach with 2 biomarkers. Am J Clin Nutr 105, 685-694. 